\title{
Characterization of air-liquid interface culture of A549 alveolar epithelial cells
}

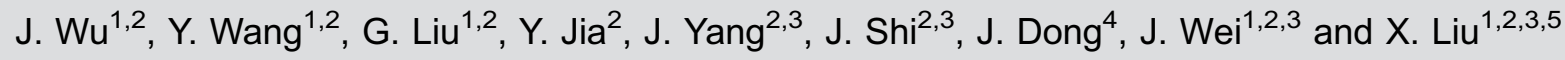 \\ ${ }^{1}$ College of Clinical Medicine, Ningxia Medical University, Yinchuan, Ningxia, China \\ ${ }^{2}$ Institute of Human Stem Cell Research, General Hospital of Ningxia Medical University, Yinchuan, Ningxia, China \\ ${ }^{3}$ Center of Laboratory Medicine, General Hospital of Ningxia Medical University, Yinchuan, Ningxia, China \\ ${ }^{4}$ Department of Pathology, Ningxia Medical University, Yinchuan, Ningxia, China \\ ${ }^{5}$ College of Life Science, Ningxia University, Yinchuan, Ningxia, China
}

\begin{abstract}
Alveolar epithelia play an essential role in maintaining the integrity and homeostasis of lungs, in which alveolar epithelial type II cells (AECII) are a cell type with stem cell potential for epithelial injury repair and regeneration. However, mechanisms behind the physiological and pathological roles of alveolar epithelia in human lungs remain largely unknown, partially owing to the difficulty of isolation and culture of primary human AECII cells. In the present study, we aimed to characterize alveolar epithelia generated from A549 lung adenocarcinoma cells that were cultured in an air-liquid interface (ALI) state. Morphological analysis demonstrated that A549 cells could reconstitute epithelial layers in ALI cultures as evaluated by histochemistry staining and electronic microscopy. Immunofluorescent staining further revealed an expression of alveolar epithelial type I cell (AECI) markers aquaporin-5 protein (AQP-5), and AECII cell marker surfactant protein C (SPC) in subpopulations of ALI cultured cells. Importantly, molecular analysis further revealed the expression of AQP-5, SPC, thyroid transcription factor-1, zonula occludens-1 and Mucin 5B in A549 ALI cultures as determined by both immunoblotting and quantitative RT-PCR assay. These results suggest that the ALI culture of A549 cells can partially mimic the property of alveolar epithelia, which may be a feasible and alternative model for investigating roles and mechanisms of alveolar epithelia in vitro.
\end{abstract}

Key words: Alveolar epithelium; A549 cells; Air-liquid interface; Alveolar epithelial type II cells; Alveolar epithelial type I cells

\section{Introduction}

Alveoli are the main terminal structures of distal airway for gas exchange and the units of lung function. The surfaces of alveoli are composed of two types of epithelial cell: alveolar type I cells (AECl) and alveolar type II (AECII) cells (1). AECl cells are applanate-shaped cells and cover $95 \%$ of the alveolar surface of the lung, although they are in fewer number than AECII cells. The major function of $\mathrm{AECl}$ cells is gas exchange, and ion and fluid balance maintenance (2). AECII cells, however, are large and cuboidal-shaped cells, and cover a much smaller percentage of alveolar surfaces. Of note, AECII cells are specialized epithelial cells for maintaining lung function and homeostasis. AECII cells can secrete active substance and regulate the metabolism of alveolar surfactant, express innate immune molecules, and regenerate and restore alveoli in response to an injury. Importantly, a subset of AECIl cells has been characterized as epithelial progenitors or stem cells with a capacity to differentiate into AECI cells or give rise to new AECII cells during alveolar injury repairs $(3,4)$.
Indeed, AECII cells are one of the most investigated cell types for understanding the physiological and pathological mechanisms of many pulmonary diseases, such as lung cancer and pulmonary fibrosis. To this end, a method for the isolation and culture of AECII cells mimicking alveolar microenvironment in vivo is becoming essential. However, this technology has not been implemented yet, partially owing to primary AECII cells losing their phenotype and expression of cell markers during the traditionally submerged culturing in vitro (5). In addition, alternative strategies using immortalized or tumor AECII cell lines also fail to fully differentiate into alveolar epithelial cell phenotypes that are seen in vivo (5). Therefore, culturing alveolar epithelial cells in submerged cell-culture conditions is an ineffective and artificial environment (6). To better mimic the in vivo environment of alveolar epithelial cells, in vitro models including 3-dimensional cultures under airflow, air-liquid interface culture, with tissue stretching and movement have been developed (4). 
However, a compelling body of studies have successfully demonstrated the isolation and in vitro culturing of AECIl cells for many species, including human, mouse, pig, and rat. Moreover, the air-liquid interface (ALI) cultures using rat and human AECIl cells demonstrated the potential of $A E C I l$ cells to differentiate into $\mathrm{AECl}$ cells in vitro (7). However, unlike the feasibility and availability of isolating and ALI-culturing human primary epithelial cells from large airway, such as tracheal, bronchi and bronchioles (8), the isolation and long-term culturing to obtain sufficient AECII for ALI culturing are difficulty and currently infeasible. Therefore, submerged cultures of immortalized or tumor AECII cell lines, such as A549 cells are currently used as models of alveolar epithelia in most in vitro studies. The objective of this study was to characterize the epithelial property of A549 cells cultured under an ALI condition.

\section{Material and Methods}

\section{Cell culture}

Human adenocarcinoma A549 (ATCC\# CCL-185) cell line was purchased from American Type Culture Collection (ATCC, USA). Cells were cultured in 1640 medium (Gibco, USA) supplemented with $10 \%$ fetal bovine serum (FBS) and 1\% penicillin-streptomycin and maintained at $37^{\circ} \mathrm{C}$ incubator in atmosphere of $5 \% \mathrm{CO}_{2}$. For generation of $\mathrm{ALI}$ cultures, membranes of Millicell inserts $(0.4-\mathrm{mm}$ pore, polycarbonate, Millipore, USA) were pre-coated with $70 \mu \mathrm{g} / \mathrm{mL}$ of type I rat tail collagen (BD Biosciences, USA), single cell suspension of A549 cells were seeded on apical surfaces of collagen-pre-coated membranes with densities of $3 \times 10^{6}$ and $0.5 \times 10^{6}$ cells per well for diameters of 30 and $12 \mathrm{~mm}$ inserts, respectively. The culture medium of the apical side was removed at $24 \mathrm{~h}$ after the seeding to establish an ALI condition. The ALI cultured cells were refreshed with medium in the bottom of insert at two-day intervals $(9,10)$.

\section{Hematoxylin and eosin histochemical staining}

After 2-weeks culturing, ALI culture inserts were fixed with $4 \%$ paraformaldehyde, and dehydrated in gradient alcohol series before they were embedded in paraffin. Sections of 4- $\mu \mathrm{m}$ thickness were employed for hematoxylin and eosin (HE) staining. The morphology of cells was observed under the Olympus BX43 light microscopy equipped with DP-73 camera (Olympus China, Shanghai, China).

\section{Immunofluorescence staining}

Immunofluorescent staining was applied to determine the expression of AECII cell marker surfactant protein $C$ (SPC) and AECl cell marker aquaporin-5 (AQP-5). The membranes of 2-week ALI cultures were fixed in filtered 4\% paraformaldehyde at room temperature for $15 \mathrm{~min}$ prior to be washed for $3 \times 3 \mathrm{~min}$ with PBS. The cells were then permeabilized with $0.2 \%$ Triton $X-100$ for 20 min at room temperature, followed by blocking with $5 \%$ normal donkey serum in PBS at room temperature for $60 \mathrm{~min}$, after which they were incubated with primary antibodies against SPC (1:1000, Merck Millipore, USA) or AQP-5 (1:500, Abcam, USA) in PBS at $4^{\circ} \mathrm{C}$ overnight. Following extensive washing for $3 \times 10$ min with PBS to remove primary antibodies, the membranes were incubated with Alexa Fluor 488-labelled donkey-anti-rabbit lgG secondary antibody (1:500, Jackson ImmunoResearch Laboratories, USA) at room temperature for $60 \mathrm{~min}$. The stained membranes were then mounted on slides with Vectashield Mounting Medium containing DAPI (Vector Laboratories, USA), and covered with a coverslip after washing in PBS for $3 \times 5 \mathrm{~min}$. Images were acquired by Leica TCS SP2 AOBS Confocal System and processed on Leica Confocal Software v.2.6.1 (Leica, Germany).

\section{Electron microscopy}

Scan electron microscopy (SEM) and transmission electronic microscopy (TEM) were employed for morphological evaluation of cell differentiation after 2 weeks postseeding. For SEM, 2-week-old ALI culture inserts were rinsed twice in PBS and fixed in 3.5\% glutaraldehyde in $0.15 \mathrm{M}$ phosphate buffer, $\mathrm{pH} 7.4$, at room temperature for $1 \mathrm{~h}$. They were then dehydrated in gradient ethanol series and treated with hexamethyldisilazane. After air-dried at room temperature, the membrane was sputter-coated with platinum/palladium prior to be visualized on a Hitachi S-3400 Microscope (Hitachi LTD, Japan) (11). For TEM, the membranes were first fixed as for SEM, followed by infiltration with Spurr resin following dehydration. Serial sections of $80 \mathrm{~nm}$ were then viewed on a Hitachi $\mathrm{H}-7000$ Electron Microscope (Hitachi LTD) $(12,13)$.

Table 1. Sequences of primer sets used in this study.

\begin{tabular}{llc}
\hline Gene & Forward primer $\left(5^{\prime}-3^{\prime}\right)$ & Reverse primer $\left(5^{\prime}-3^{\prime}\right)$ \\
\hline SP-C & CCTGAAACGCCTTCTTATCG & CTCCAGAACCTACTCCGTGT \\
AQP-5 & ATGGTGGTGGAGCTGATTCT & GGTGACAGACAGGCCAATG \\
ZO-1 & ATGACTCCTGACGGTTGGTC & CACAGTTTGCTCCAACGAGA \\
MUC5B & CCACAGCTACCAGCGTTACA & TGGAGTAGAGGAGGGTGTGG \\
TTF-1 & GGCATTGAGAGTGCAGACAA & GGGCAATGTTCCCACCAATG \\
$\beta$-actin & CTCTTCCAGCCTTCCTTCCT & AGCACTGTGTTGGCGTACAG \\
\hline
\end{tabular}




\section{Immunoblotting analysis}

Whole cell extracts were prepared homogenizing cells in RIPA buffer for $3.5 \mathrm{~h}$ on ice. The cell lysate was centrifuged at $12,000 \mathrm{~g}$ for $30 \mathrm{~min}$ at $4^{\circ} \mathrm{C}$ for clarifying. The resultant supernatant of lysates $(45 \mu \mathrm{g})$ were resolved on $10 \%$ sodium dodecyl sulfate polyacrylamide gel (SDSPAGE) and transferred to PVDF membranes (Millipore). The membranes were blocked with $5 \%$ fat-free dry milk in PBS containing $0.1 \%$ Tween-20 for $1 \mathrm{~h}$ at room temperature, and probed with primary antibodies to proteins of interest at $4^{\circ} \mathrm{C}$ overnight. Then the membranes were incubated with appropriate horseradish peroxidase labeled secondary antibodies for $1 \mathrm{~h}$ at room temperature. The blots were then developed using the enhanced Western Bright ECL reagent (Advansta Inc., USA). The levels of protein expression were semi-quantified by absorbance using ImageJ Software version 1.46 (http://rsb.info.nih.gov/ij/). The ratio between the net intensity of each sample divided by the $\beta$-actin internal control was calculated as densitometric arbitrary units (AU) which served as an index of relative expression of a protein of interest $(14,15)$. The primary antibodies used in this study were rabbit antiSP-C (1:1000, Merck Millipore, USA), rabbit anti-AQP-5 (1:1000, Abcam, USA), mouse anti-thyroid transcription factor-1 (TTF-1; 1:1000, Thermo Fisher Scientific, UK), rabbit anti-zonula occludens-1 (ZO-1; 1:1000, Zymed Lab Inc., USA), rabbit anti-Mucin 5B (MUC5B; 1:1000, Santa Cruz Biotech, USA) and rabbit anti- $\beta$-actin (1:1000, Santa Cruz Biotech).

\section{RNA isolation and real-time quantitative RT-PCR}

The total RNA from differently treated cells was purified using Trizol reagent per manufacturer's instruction (Invitrogen, USA). The quality of RNA was assayed by calculation of the RNA integrity number (RIN). High quality RNA (RIN value greater than 9.0) was used for reverse transcription of first-strand cDNA synthesis by reverse transcription using M-MLV reverse transcriptase (TransGen Biotech, China), and the transcript expression of the gene of interest was amplified using TransStart Tip Green qPCR SuperMix (TransGen Biotech) in LightCycler 480 II PCR system (Roche Diagnostics, Switzerland). The thermal cycling condition for PCR amplification was $95^{\circ} \mathrm{C}$ for $30 \mathrm{~s}, 40$ cycles of $95^{\circ} \mathrm{C}$ for $5 \mathrm{~s}, 60^{\circ} \mathrm{C}$ for $20 \mathrm{~s}$, and $72^{\circ} \mathrm{C}$ for $20 \mathrm{~s}$, followed by $40^{\circ} \mathrm{C}$ for $20 \mathrm{~min}$. The primer sets used for RT-PCR were designed and synthesized in Shanghai Sangon Biotech Inc. (China) by bioinformatics tools using available mRNA sequences. The sequences of primers are listed in Table 1. The internal controls were always included to normalize each reaction with respect to RNA integrity, sample loading and inter-PCR variations. The relative expression ratio was calculated from the realtime PCR efficiencies and the crossing point deviation of a given gene $v s$. $\beta$-actin. In each independent experiment, the mean gene expression ratios obtained with regular submerged cultures were given a value of 1 (fold).
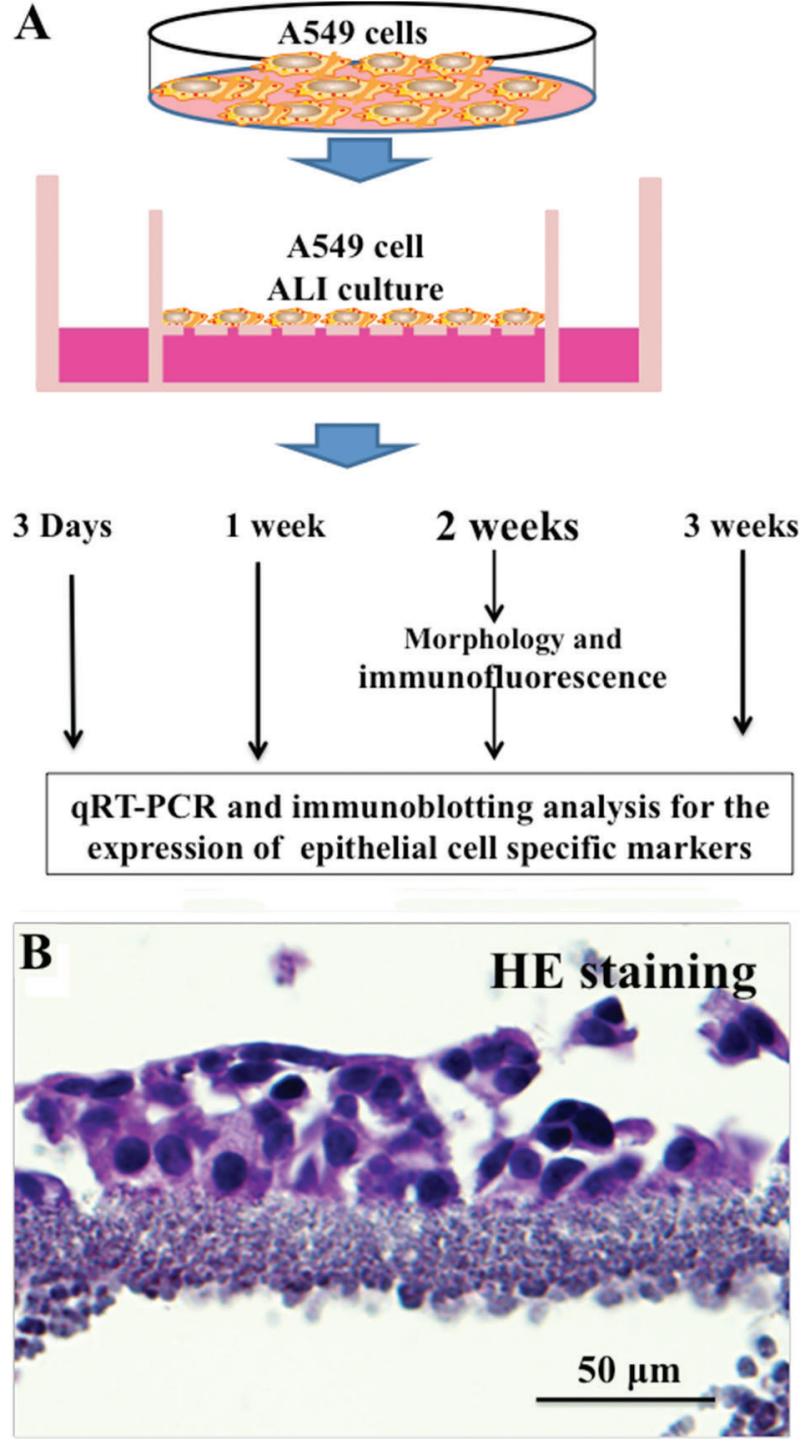

Figure 1. The experimental process and histochemical staining. $A$, Experimental design and process. A549 cells were seeded on collagen pre-coated membranes of Millicell inserts, and cultured under an air-liquid interface (ALI) state for the indicated times. Membranes were then collected for morphological and molecular analysis. B, Morphological evaluation of a 2-week-old A549 epithelial cell ALI culture by hematoxylin and eosin staining, showing the epithelial layer of A549 cells grown on the membranes.

The specificity of PCR was determined by sequencing of the PCR products.

\section{Statistical analysis}

All data collected in this study was obtained from at least three independent experiments for each condition. SPSS19.0 analysis software (USA) and PRISM 5 (Graphpad, USA) were used for the statistical analysis. Statistical evaluation of the data was performed by one-way ANOVA 

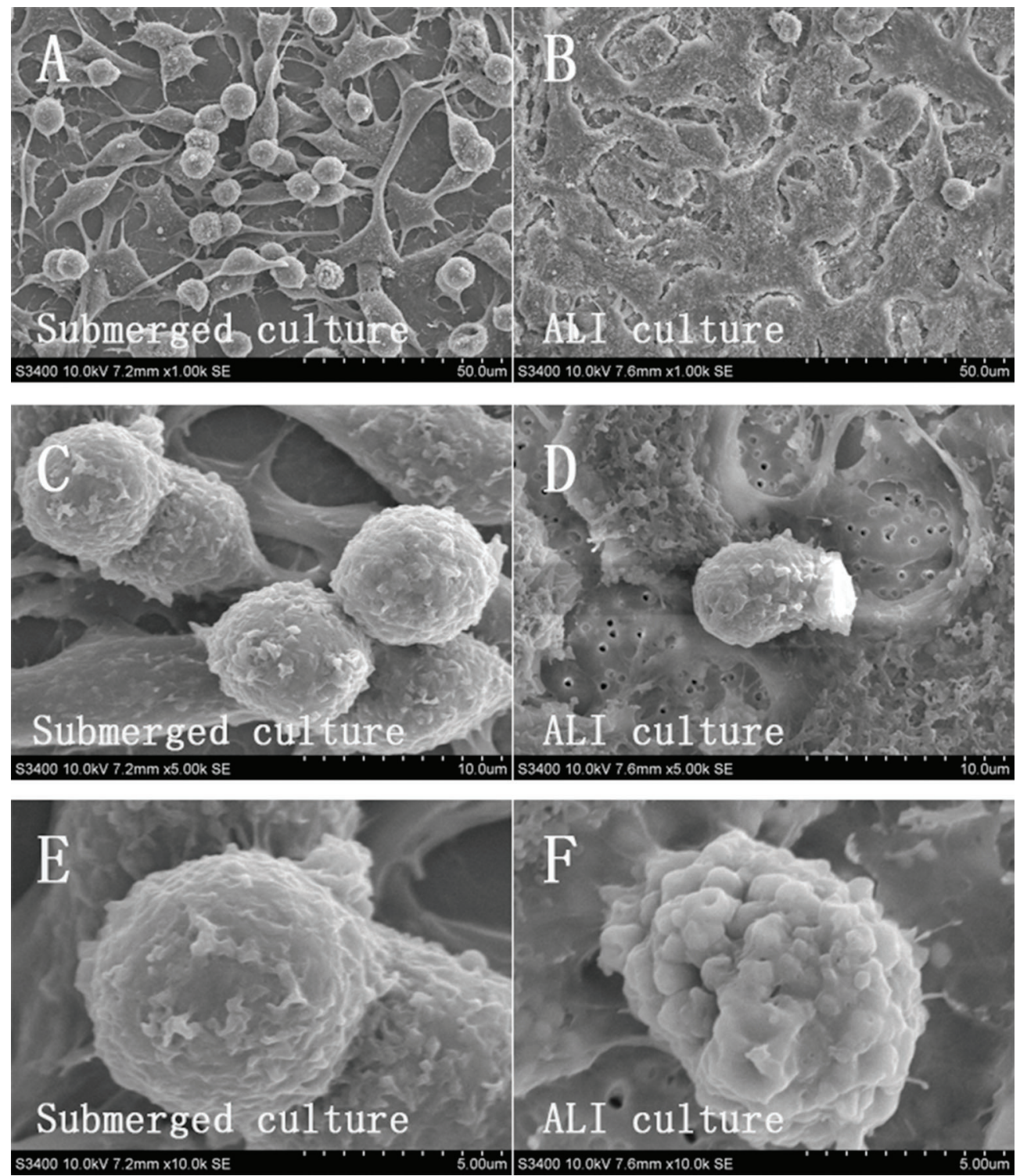

Figure 2. Scanning electronic microscopy (SEM) of air-liquid interface (ALI) of 2-week-old A549 ALI cultures and logarithmic phase of submerged cultures. $A, C$, and $E$, Different magnifications of submerged cultures showing a morphology of inerratic shapes with smooth surfaces; $B, D$, and $F$, different magnifications of ALI cultures exhibiting anomalous shapes and rough cell surfaces with abundant secretion on the surface of culture. Magnification: $A$ and $B=50 \mu \mathrm{m} ; C$ and $D=10 \mu \mathrm{m} ; E$ and $F=5.0 \mu \mathrm{m}$.

when more than two groups were compared with a single control, and $t$-test was employed for comparison of differences between two groups. $\mathrm{P}<0.05$ was considered as a statistically significant difference. Data are reported as means \pm SD.

\section{Results}

\section{Morphological analysis of A549 cell ALI cultures}

In order to characterize ALI culture of A549 cells, A549 cells were seeded on collagen-pre-coated membranes of
Millicell inserts and cultured in an air-liquid interface phase. The cells were evaluated for alveolar epithelial cell phenotypes by ascertaining their morphology and the expression of cell type-specific markers at different time points using appropriate assays (Figure 1A). Morphological analysis revealed the formation of an intact epithelial cell layer on the membrane as seen by $\mathrm{HE}$ staining (Figure 1B). The conventionally submerged culture cells exhibited inerratic shapes with smooth surface, but ALI cultured cells displayed anomalous shapes and rough cell surfaces with abundant secretions observed under a 

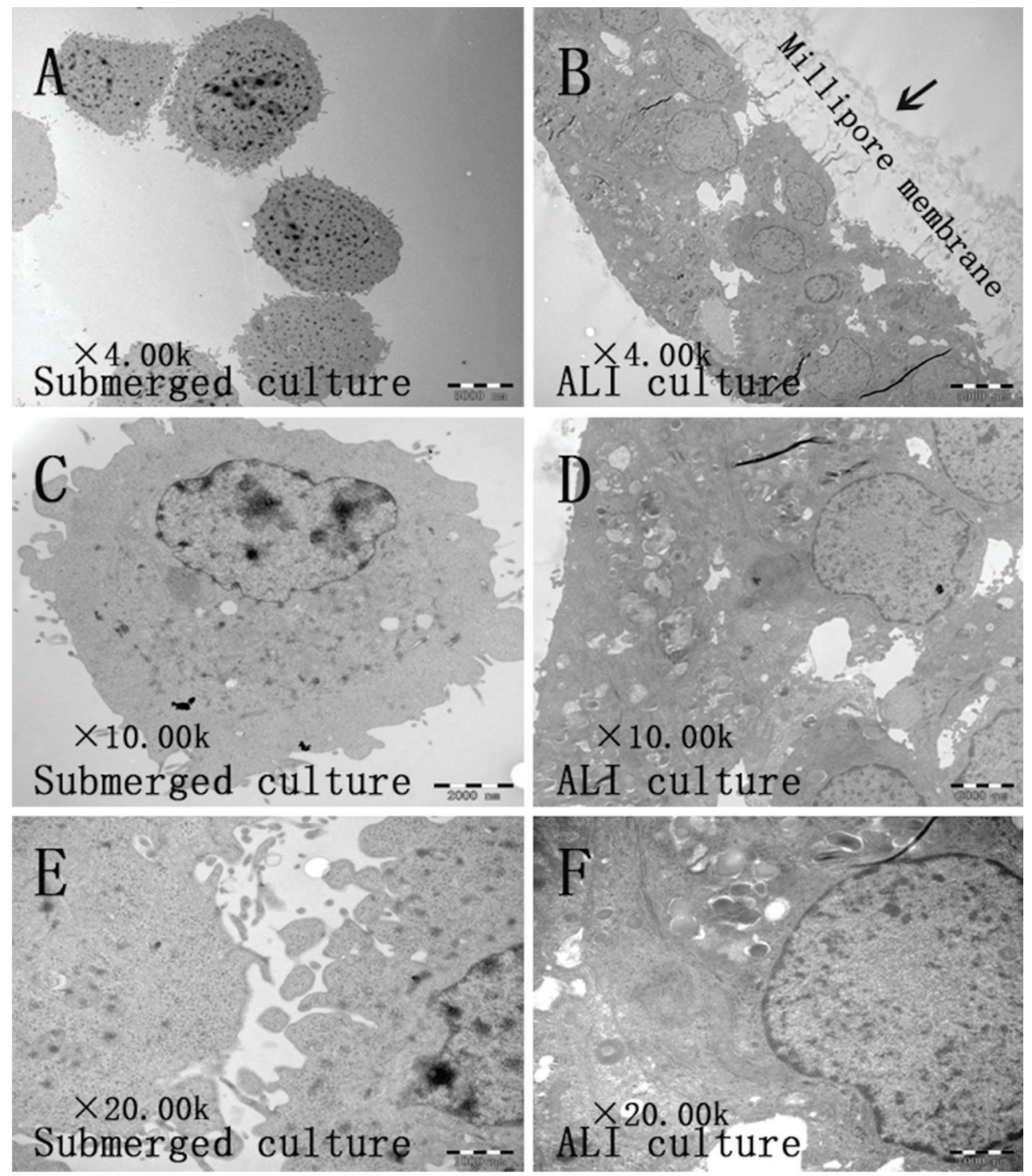

Figure 3. Transmission electron microscopy (TEM) of air-liquid interface (ALI) cultures and logarithmic phase of submerged cultures of A549 cells. $A, C$, and $E$, Different magnifications of submerged cultures showing a morphology of clear border and organelles and lack of obvious microvilli, although they had AECl-like outward appearance in some extent; $B, D$, and $F$, different magnifications of $A L I$ cultured cells displaying a more compacted morphology with clear cell junction and abundant mucus on the apical surface. Magnification bars: $A$ and $B=5000 \mathrm{~nm} ; C$ and $D=2000 \mathrm{~nm} ; E$ and $F=1000 \mathrm{~nm}$.

scanning electron microscopy (Figure 2). Morphological analysis using transmission electron microscopy further revealed that the submerged cell cultures exhibited clear border and organelles, and were lacking obvious microvilli, although they had $\mathrm{AECl}$-like outward appearance in some extent (16) (Figure 3A, C and E). However, the ALI cultured cells were more compacted with clear cell junctions and abundant mucus on the apical surface (Figure 3B, D and F).

\section{Immunological characterization of A549 cell \\ ALI cultures}

To further characterize A549 cells in ALI cultures, immunofluorescent staining assay was used to determine the expression of cell-specific markers of $\mathrm{AECl}$ and $\mathrm{AECII}$ cells. As expected, the expression of $\mathrm{AECl}$ cell specific marker AQP-5 and AECII cell marker SP-C were observed on the cytomembrane and in the cytoplasm of subset cells, respectively (Figure 4). 

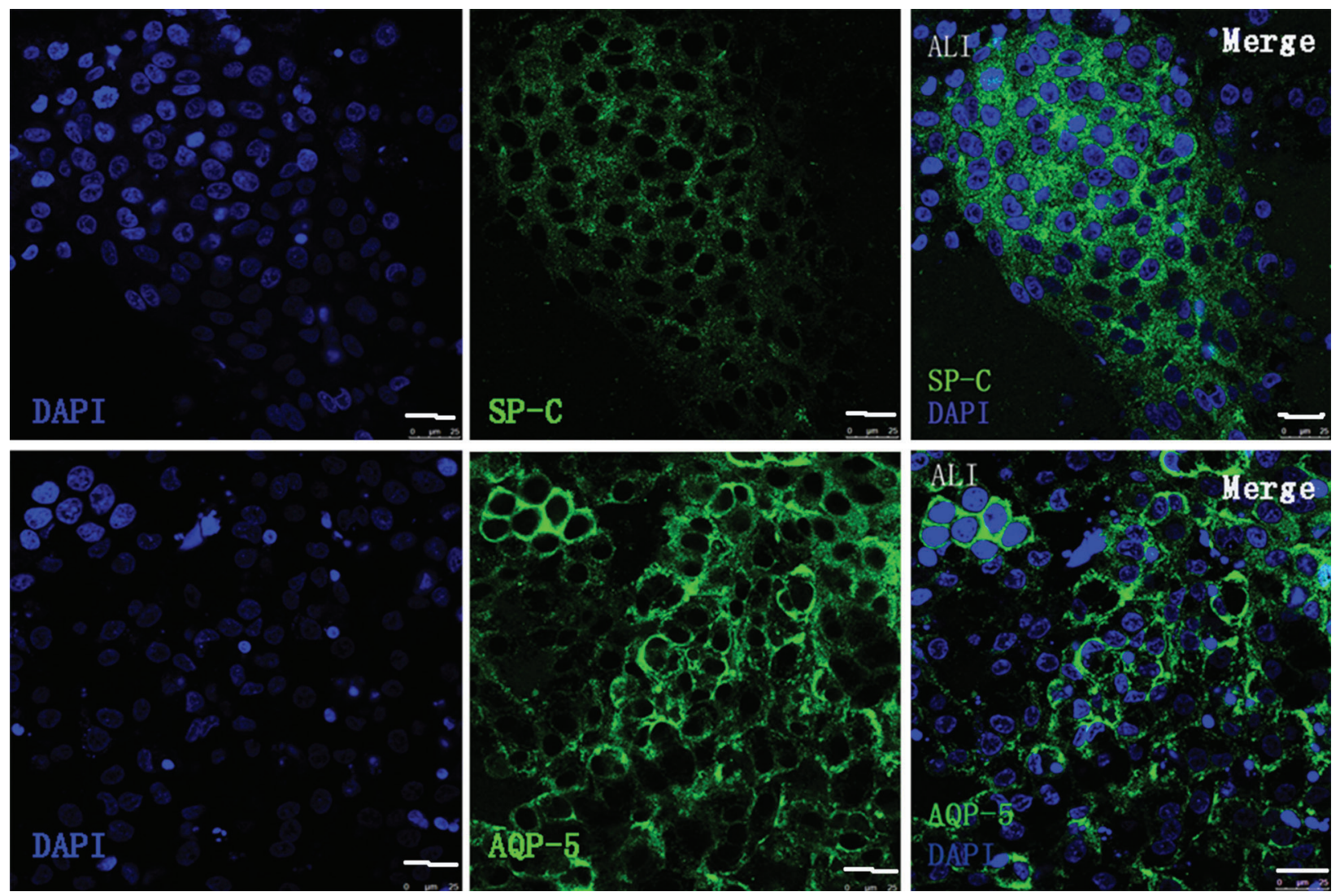

Figure 4. Expression of alveolar epithelial cell type markers determined by immunofluorescent staining. Two-week-old membranes of A549 air-liquid interface (ALI) cultures were used for determination of the expression of $\mathrm{AECl}$ cell specific marker aquaporin-5 (AQP-5) and AECII cell marker surfactant protein C (SP-C) by immunofluorescent staining assay. The expression of both of AQP-5 and SPC was observed on the cytomembrane and in the cytoplasm of distinct subset cells, respectively. Magnification bars: $25 \mu \mathrm{m}$.

\section{Molecular characterization of A549 cell ALI cultures}

AECII cells were reported to lose phenotype markers over time when they were cultured in monolayer (17), but they might be able to express cell type-specific markers in an ALI interface culture (4). Therefore, we sought to analyze the expression of epithelial cell markers in both transcriptional and translational levels by qRT-PCR and immunoblotting assays, respectively. Indeed, transcriptional analysis revealed that significantly more transcripts of SPC, AQP-5, TTF-1, MUC5B and ZO-1 were detected in ALI cultures compared to submerged cultures, particularly in the 2-week-old ALI cultures (Figure 5). However, a dynamic change in proteins of epithelial markers was observed in A549 cell ALI cultures as determined by the immunoblotting assay. The overall SPC protein was moderately increased in ALI cultures in comparison with submerged cultures. Consistently, the $\mathrm{AECl}$ cell marker proteins AQP5 and TTF-1 were also increased in ALI cultures compared to submerged cultures. An increased mucin 5B (MUC5B) was also observed in ALI cultures.
Interestingly, the epithelial tight junction protein ZO-1 was detected in both ALI cultures and submerged cultures, except it failed to be detected in ALI cultures of 3 days. All the above examined proteins of interest were found increased in ALI cultures of 1 and 2 weeks, compared with submerged cell cultures (Figure 6), suggesting that 1-2 weeks was the best window for the alveolar property of the A549 ALI culture model. Together with the aforementioned morphological data, this result suggested that A549 cells might partially maintain alveolar epithelial cell properties in ALI state.

\section{Discussion}

The purpose of this study was to characterize alveolar epithelial properties of a three-dimensional (3D) ALI culture of A549 cells. Subpopulations of ALI cultured A549 cells exhibited abilities to partially express cell type-specific markers of $\mathrm{AECl}$ and $\mathrm{AECI}$ cells. The ALI culture model has several advantages compared to submerged cultures 

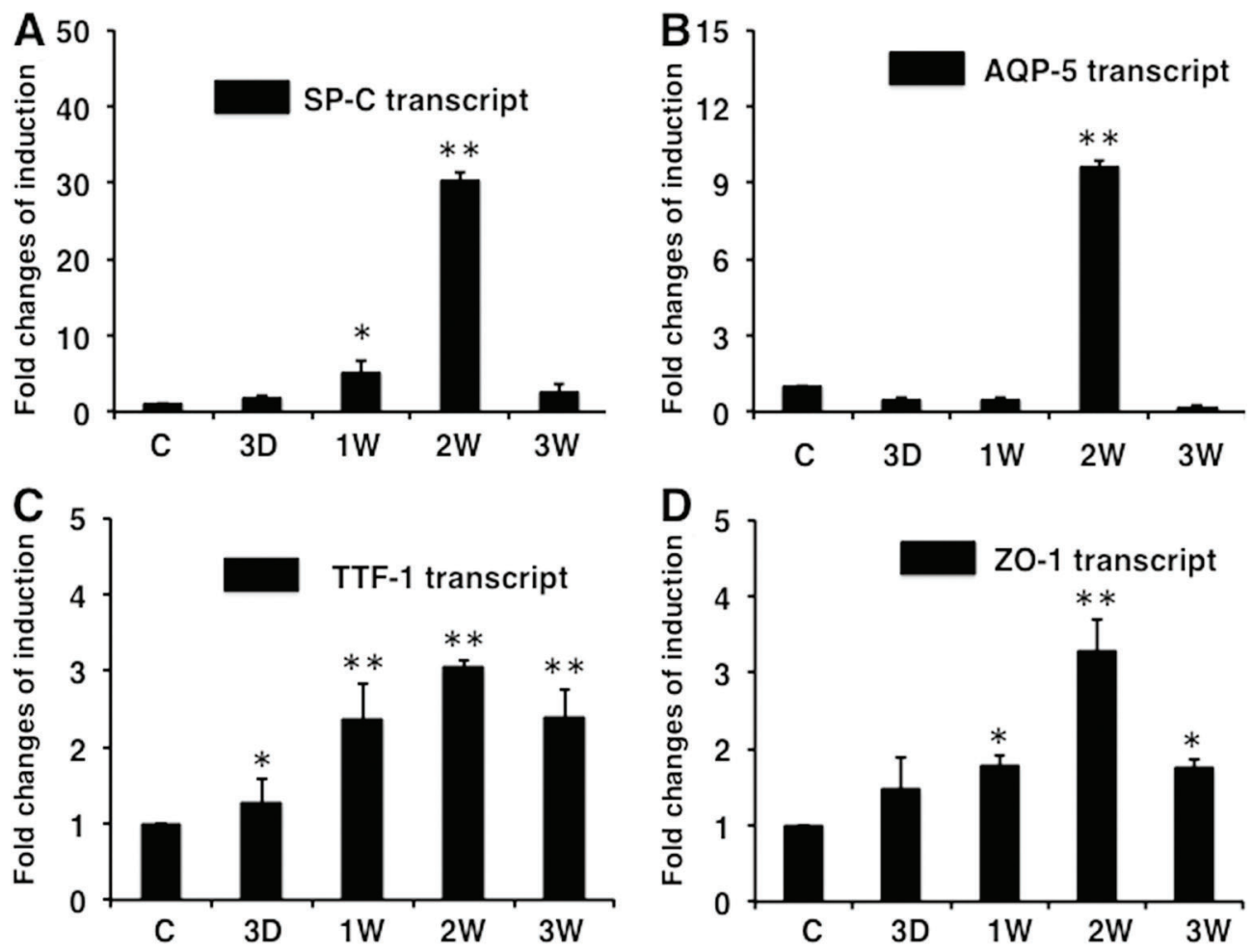

Figure 5. Transcripts of several key genes of interest in A549 cell air-liquid interface (ALI) cultures at 3 days, 1 week, 2 weeks, and 3 weeks were examined by a qRT-PCR assay. A significantly induced expression of SP-C $(A)$, AQP-5 (B), TTF-1 (C), and ZO-1 (D) mRNAs were observed in ALI cultures compared to submerged cultures of A549 cells (control, C), particularly in the 2-week-old cultures. Data are reported as means $\pm S D$ from 3 independent experiments $(n=9)$., D: days; W: week(s). ${ }^{*} P<0.05$ and ${ }^{* *} P<0.01$ compared to the submerged culture control (ANOVA).

of immortalized or tumor cell lines. Therefore, ALI epithelial culture of A549 cells might be a potentially useful and feasible in vitro model for investigating the physiological and pathological roles of alveolar epithelial cells in human lungs. In addition, the ALI culture of A549 cells would better mimic alveolar epithelia compared to the submerged A549 cell cultures.

AECII cells have a potency to give rise to new AECII cells and differentiate into $\mathrm{AECl}$ cells during alveolar injury repair and regeneration, therefore they have been considered progenitor cells in the alveoli $(3,4,18)$. Previous studies have demonstrated that primary AECII cultured under a conventional or submerged cell culture condition showed a loss of AECII cell phenotype and of the surfactant synthesis capacity, and cell morphology alteration (19). A compelling body of studies has demonstrated the capacity of primary airway epithelial cells to reconstitute airway epithelia of many species in ALI culture models, including human (10), sheep (11), bovine (14), mouse (13), non-human primate (20), pig and ferret (9). Primary airway epithelial cells isolated from large airways, such as the trachea and bronchus of different species, are able to fully differentiate into various epithelial cell types in ALI culture model, which is considered an organotypic model (21). Despite AECII cells isolated from bovine (22), human $(1,23,24)$, pig $(25,26)$, mouse $(27)$, and rat (7) lungs exhibited the ability to maintain their cell phenotype in an ALI culture model $(7,24)$, it remains a great challenge to isolate or culture sufficient AECII cells for generation of ALI cultures. Therefore, there is a need to develop an alternative model for investigating roles and mechanisms of alveolar epithelia. In the present study, the characterization of A549 cells cultured in an ALI phase was examined. The results showed that the expression of alveolar epithelial type I cell markers AQP-5 and TTF-1 was significantly increased in ALI cultures as compared with submerged cultured A549 cells. In addition, mucin 5B and ZO-1 were determined in the ALI cultures, but the expression of AECII cell marker SPC was only moderately increased.

A previous study has suggested that dipalmitoyl phosphatidyl choline-containing multilamellar bodies (MLB) are ultrastructural hallmark of AECII cells (28). The other hallmark of AECII cells includes lipids, which are major components of pulmonary surfactant that are able to prevent alveoli from collapse by reducing alveolar surface tension at 

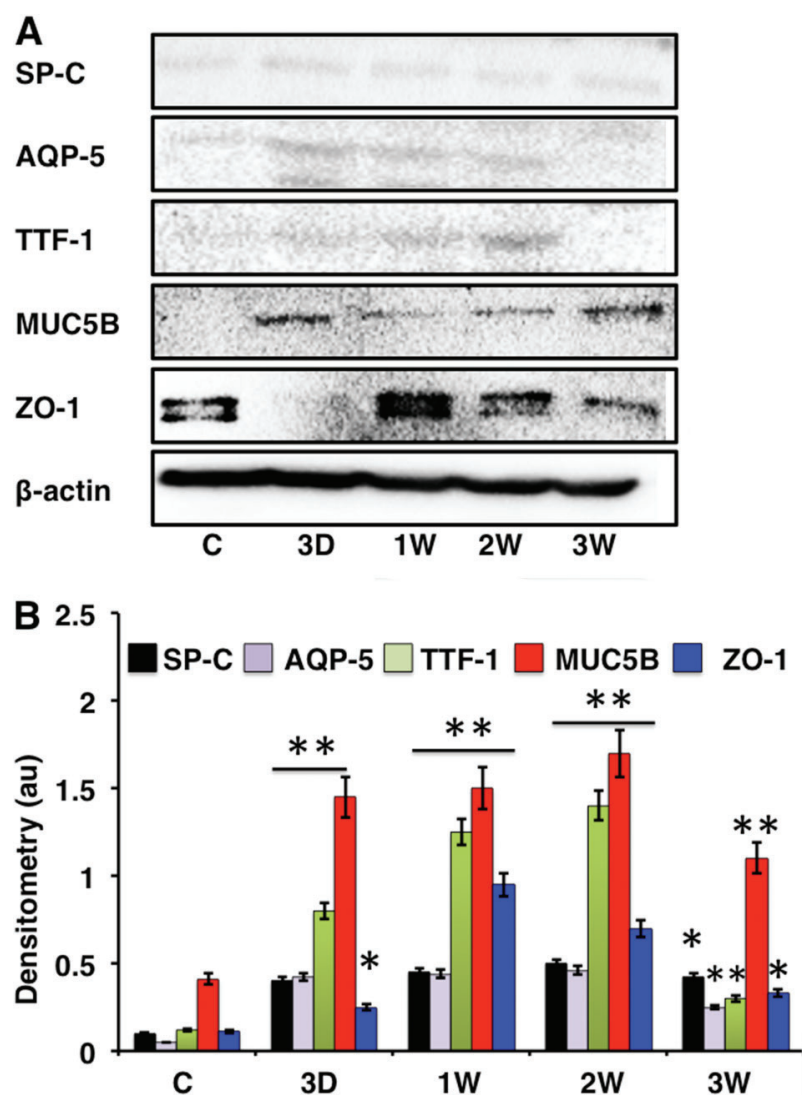

Figure 6. Protein of several key cell-specific markers of alveolar epithelial cells in A549 cell air-liquid interface (ALI) cultures at 3 days, 1 week, 2 weeks and 3 weeks were examined by an immunoblotting assay using specific antibodies. $A$, Representative blots of immunoblotting assay showing significantly higher levels of AECl cell type markers AQP-5, TTF-1, and epithelial cell proteins MUC5B and ZO-1 observed in ALI cultures compared to submerged cultures of A549 cells (control, C). However, AECII cell type-specific marker SPC was only moderately increased in ALI cultures compared to submerged cultures of A549 cells. $B$, Densitometry values are reported as fold-change for SPC, AQP-5, TTF-1, MUC5B and ZO-1 proteins over $\beta$-actin. Data in $B$ are reported as means \pm SD from 3 independent experiments $(n=3)$. D: days; W: week(s). ${ }^{*} P<0.05$ and ${ }^{* *} P<0.01$ compared to the submerged culture control (ANOVA).

the end of expiration (28). In addition, lysosomes have also been thought as an origin of MLB, and a long-term culture

\section{References}

1. Fujino $\mathrm{N}$, Kubo $\mathrm{H}$, Suzuki T, Ota $\mathrm{C}$, Hegab AE, He M, et al. Isolation of alveolar epithelial type ii progenitor cells from adult human lungs. Lab Invest 2011; 91: 363-378, doi: 10.1038/labinvest.2010.187.

2. O'Reilly MA. Giving new identities to alveolar epithelial type i cells. Am J Respir Cell Mol Biol 2017; 56: 277-278, doi: 10.1165/rcmb.2016-0383ED. of A549 cells may lead the accumulation of lysosomes (28). In agreement with these findings, the morphological analysis of electron microscopy also revealed a large number of lipid droplets and lysosomes in the cytoplasm in the 2-week-old ALI culture of A549 cells (28). Equally noteworthy, A549 cells are adenocarcinoma cells with unlimited capacity of proliferation, but limited differentiation, although they have been widely used as an AECII cell models in vitro (29). In line with this, we also noted that a higher level of alveolar epithelial cell markers was determined in 1- to 2-week-old ALI cultures of A549 cells rather than cultures of an earlier or later time period. We reasoned that A549 cells could differentiate into alveolar epithelial cell types at some extent in early time points, but they might retain their carcinoma cell properties along with a reduced capacity of differentiation in a long-term ALI culture. However, the exact mechanism of this phenotype of cultures need to be further investigated. The 1- to 2-week window for the best alveolar properties of A549 ALI culture model limits the use of this model in studies that a long-term follow up is required. In addition, despite an increased expression of $\mathrm{AECl}$ cell type markers AQP-5 and TTF-1 being observed in ALIcultured A549 cells, AECl cells have not been morphologically identified. This also implies a limitation of ALI cultures generated from A549 cells and a necessity of primary AECII cells for generation of alveolar epithelial model in vitro.

Collectively, in the present report, an ALI epithelial culture model of A549 cells was characterized. The results revealed an induced expression of alveolar epithelial specific cell markers AQP-5 and SPC in ALI cultured A549 cells, indicating partial alveolar epithelial cell properties. These results thus suggest that the ALI-cultured A549 cells may be a feasible and alternative model for investigating roles and mechanisms of alveolar epithelia in vitro.

\section{Acknowledgments}

This work was supported by grants from the National Natural Science Foundation of China (Nos. 31472191 and 81460247), a grant from the program of the First-class Discipline Construction of Clinical Medicine of Ningxia Medical University, and a grant from the Plan of Postgraduate Education Innovation, Discipline Construction Project of Ningxia, China (2017, No. YXW2017014).
3. Uhl FE, Vierkotten S, Wagner DE, Burgstaller G, Costa R, Koch I, et al. Preclinical validation and imaging of wntinduced repair in human 3d lung tissue cultures. Eur Respir J 2015; 46: 1150-1166, doi: 10.1183/09031936.00183214.

4. Marcinkiewicz MM, Baker ST, Wu J, Hubert TL, Wolfson MR. A novel approach for ovine primary alveolar epithelial type ii cell isolation and culture from fresh and cryopreserved 
tissue obtained from premature and juvenile animals. PLoS One 2016; 11: e0152027, doi: 10.1371/journal.pone. 0152027.

5. Grek CL, Newton DA, Qu Y, Wen X, Spyropoulos DD, Baatz JE. Characterization of alveolar epithelial cells cultured in semipermeable hollow fibers. Exp Lung Res 2009; 35: 155-174, doi: 10.1080/01902140802495870.

6. Lenz AG, Karg E, Brendel E, Hinze-Heyn H, Maier KL, Eickelberg $\mathrm{O}$, et al. Inflammatory and oxidative stress responses of an alveolar epithelial cell line to airborne zinc oxide nanoparticles at the air-liquid interface: A comparison with conventional, submerged cell-culture conditions. Biomed Res Int 2013; 2013: 652632, doi: 10.1155/2013/ 652632.

7. Dobbs LG, Pian MS, Maglio M, Dumars S, Allen L. Maintenance of the differentiated type ii cell phenotype by culture with an apical air surface. Am J Physiol 1997; 273 (Part 1): L347-L354.

8. Emura $M$, Aufderheide M. Challenge for $3 d$ culture technology: Application in carcinogenesis studies with human airway epithelial cells. Exp Toxicol Pathol 2016; 68: 255-261, doi: 10.1016/j.etp.2016.02.002.

9. Liu X, Luo M, Guo C, Yan Z, Wang Y, Engelhardt JF. Comparative biology of raav transduction in ferret, pig and human airway epithelia. Gene Ther 2007;14: 1543-1548, doi: 10.1038/sj.gt.3303014.

10. Liu X, Driskell RR, Engelhardt JF. Stem cells in the lung. Methods Enzymol 2006; 419: 285-321, doi: 10.1016/S00766879(06)19012-6.

11. Xue D, Ma Y, Li M, Li Y, Luo H, Liu X, et al. Mycoplasma ovipneumoniae induces inflammatory response in sheep airway epithelial cells via a myd88-dependent tir signaling pathway. Vet Immunol Immunopathol 2015; 163: 57-66, doi: 10.1016/j.vetimm.2014.11.008.

12. Kemp SJ, Thorley AJ, Gorelik J, Seckl MJ, O'Hare MJ, Arcaro A, et al. Immortalization of human alveolar epithelial cells to investigate nanoparticle uptake. Am J Respir Cell Mol Biol 2008; 39: 591-597, doi: 10.1165/rcmb.20070334OC.

13. Liu X, Yan Z, Luo M, Engelhardt JF. Speciesffspecific differences in mouse and human airway epithelial biology of recombinant adeno-associated virus transduction. $\mathrm{Am} \mathrm{J}$ Respir Cell Mol Biol 2006; 34: 56-64, doi: 10.1165/rcmb. 2005-01890C.

14. Ma Y, Han F, Liang J, Yang J, Shi J, Xue J, et al. A speciesspecific activation of toll-like receptor signaling in bovine and sheep bronchial epithelial cells triggered by mycobacterial infections. Mol Immunol 2016; 71: 23-33, doi: 10.1016/ j.molimm.2016.01.004.

15. Yang J, Zhang K, Wu J, Shi J, Xue J, Li J, et al. Wnt5a increases properties of lung cancer stem cells and resistance to cisplatin through activation of wnt5a/pkc signaling pathway. Stem Cells Int 2016; 2016: 1690896, doi: 10.1155/ 2016/1690896.

16. Lu HY, Shao GB, Li WB, Wang H. Effects of hyperoxia on transdifferentiation of primary cultured typeii alveolar epithelial cells from premature rats. In vitro Cell Dev Biol Anim 2011; 47: 64-72, doi: 10.1007/s11626-010-9360-9.
17. Mason RJ, Leslie CC, Voelker DR. Alveolar type ii epithelial cells in primary culture. Curr Probl Clin Biochem 1983; 13: 24-34.

18. Evans MJ, Cabral LJ, Stephens RJ, Freeman G. Transformation of alveolar type 2 cells to type 1 cells following exposure to no2. Exp Mol Pathol 1975; 22: 142-150, doi: 10.1016/0014-4800(75)90059-3.

19. Lin S, Akinbi HT, Breslin JS, Weaver TE. Structural requirements for targeting of surfactant protein $b(s p-b)$ to secretory granules in vitro and in vivo. J Biol Chem 1996; 271: 19689-19695, doi: 10.1074/jbc.271.33.19689.

20. Liu X, Luo M, Trygg C, Yan Z, Lei-Butters DC, Smith Cl, et al. Biological differences in raav transduction of airway epithelia in humans and in old world non-human primates $\mathrm{Mol}$ Ther 2007;15: 2114-2123.

21. $\mathrm{Xu} \mathrm{W}$, Janocha AJ, Leahy RA, Klatte R, Dudzinski D, Mavrakis LA, et al. A novel method for pulmonary research: Assessment of bioenergetic function at the air-liquid interface. Redox Biol 2014; 2: 513-519, doi: 10.1016/j.redox. 2014.01.004.

22. Su F, Liu X, Liu G, Yu Y, Wang Y, Jin Y, et al. Establishment and evaluation of a stable cattle type ii alveolar epithelial cell line. PLoS One 2013; 8: e76036, doi: 10.1371/journal.pone. 0076036

23. Bove PF, Grubb BR, Okada SF, Ribeiro CM, Rogers TD, Randell SH, et al. Human alveolar type ii cells secrete and absorb liquid in response to local nucleotide signaling. J Biol Chem 2010; 285: 34939-34949, doi: 10.1074/jbc.M110. 162933.

24. Bove PF, Dang H, Cheluvaraju C, Jones LC, Liu X, O'Neal WK, et al. Breaking the in vitro alveolar type ii cell proliferation barrier while retaining ion transport properties. $A m \mathrm{~J}$ Respir Cell Mol Biol 2014; 50: 767-776, doi: 10.1165/rcmb. 2013-00710C.

25. Li X, Comellas AP, Karp PH, Ernst SE, Moninger TO, Gansemer ND, et al. Cftr is required for maximal transepithelial liquid transport in pig alveolar epithelia. $A m J$ Physiol Lung Cell Mol Physiol 2012; 303: L152-L160, doi: 10.1152/ajplung.00116.2012.

26. Steimer A, Laue M, Franke $H$, Haltner-Ukomado $E$, Lehr $\mathrm{CM}$. Porcine alveolar epithelial cells in primary culture: Morphological, bioelectrical and immunocytochemical characterization. Pharm Res 2006; 23: 2078-2093, doi: 10.1007/ s11095-006-9057-7.

27. Messier EM, Mason RJ, Kosmider B. Efficient and rapid isolation and purification of mouse alveolar type ii epithelial cells. Exp Lung Res 2012; 38: 363-373, doi: 10.3109/ 01902148.2012 .713077$.

28. Cooper JR, Abdullatif MB, Burnett EC, Kempsell KE, Conforti $\mathrm{F}$, Tolley $\mathrm{H}$, et al. Long term culture of the a549 cancer cell line promotes multilamellar body formation and differentiation towards an alveolar type ii pneumocyte phenotype. PLoS One 2016; 11: e0164438, doi: 10.1371/ journal.pone. 0164438 .

29. Swain RJ, Kemp SJ, Goldstraw P, Tetley TD, Stevens MM. Assessment of cell line models of primary human cells by raman spectral phenotyping. Biophys $J$ 2010; 98: 17031711, doi: 10.1016/j.bpj.2009.12.4289. 